\title{
Frequency-shifted sources for Terahertz-driven linear electron acceleration
}

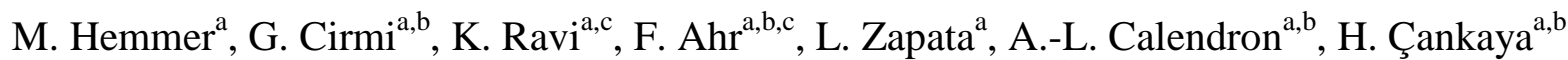 \\ S. W. Jolly ${ }^{\mathrm{a}, \mathrm{d}, \mathrm{e}}$, V. Leroux ${ }^{\mathrm{a}, \mathrm{d}, \mathrm{e}}$, T. Eichner ${ }^{\mathrm{a}, \mathrm{e}}$, H. Ishizuki ${ }^{\mathrm{f}}$, T. Taira ${ }^{\mathrm{f}}$, N. Matlis ${ }^{\mathrm{a}}$, A. R. Maier ${ }^{\mathrm{a}, \mathrm{e}}$, F.X. \\ Kärtner ${ }^{\mathrm{a}, \mathrm{b}, \mathrm{c}, \mathrm{e}}$ \\ ${ }^{\mathrm{a} C e n t e r ~ f o r ~ F r e e-E l e c t r o n ~ L a s e r ~ S c i e n c e, ~ D e u t s c h e s ~ E l e k t r o n e n ~ S y n c h r o t r o n ~(D E S Y), ~ N o t k e s t r a ß e ~}$ \\ 85, 22607 Hamburg, Germany \\ ${ }^{\mathrm{b}}$ The Hamburg Center for Ultrafast Imaging, Luruper Chaussee 149, 22607 Hamburg, Germany \\ ${ }^{\mathrm{c}}$ Department of Electrical Engineering and Computer Science, and Research Laboratory of \\ Electronics, Massachusetts Institute of Technology, 77 Massachusetts Avenue, Cambridge, \\ Massachusetts 02139, USA \\ ${ }^{\mathrm{d}}$ Institute of Physics of the ASCR, ELI-Beamlines project, Na Slovance 2, 18221 Prague, Czech \\ Republic \\ ${ }^{\mathrm{e}}$ Department of Physics, University of Hamburg, Hamburg, 22761, Germany \\ ${ }^{\mathrm{f}}$ Institute for Molecular Science, National Institutes of Natural Science, Myodaiji, Okazaki 444-8585, \\ Japan
}

\begin{abstract}
The generation of THz-frequency radiation via nonlinear parametric frequency down-conversion has long been driven by the spectroscopy and imaging communities. As a result, little efforts have been undertaken toward the generation of high energy THz-frequency pulses. THz-frequency radiation has however recently been identified has a promising driver for strong-field physics and an emerging generation of compact particle accelerators. These accelerators require $\mathrm{THz}$ frequency pulses with energies in the multi-millijoule range therefore demanding orders of magnitude improvements from the current state-of-the-art.

Much can be gained by improving the intrinsically low efficiency of the down-conversion process while still resorting to existing state-of-the-art lasers. However, the fundamental Manley-Rowe limit caps the efficiency of parametric downconversion from $1-\mu \mathrm{m}$ wavelength lasers to sub-THz frequency to the sub-percent range.

We present methods that promise boosting the $\mathrm{THz}$ radiation yield obtained via parametric down-conversion beyond the Manley-Rowe limit. Our method relies on cascaded nonlinear three-wave mixing between two spectrally neighboring laser pulses in periodically poled Lithium Niobate. Owing to favorable phase-matching, the down-conversion process avalanches, resulting in spectral broadening in the optical domain. This allows in-situ coherent multiplexing of multiple parametric down-conversion stages within a single device and boosting the efficiency of the process beyond the ManleyRowe limit. We experimentally demonstrated the concept using either broadband, spectrally chirped optical pulses from a Joule-class laser or using two narrowband lasers with neighboring wavelengths. Experimental results are backed by numerical simulations that predict conversion efficiencies from $1 \mu \mathrm{m}$ to sub-THz radiation in the multi-percent range.
\end{abstract}

Keywords: THz generation, multi-cycle, narrowband, THz driven FEL, lithium niobate, cascaded optical parametric amplification, THz particle accelerator, difference frequency generation

\section{INTRODUCTION}

Highly brilliant X-ray sources are in demand for a growing number of applications ranging from fundamental physics, chemistry and biology to industrial applications. Brilliant and highly coherent X-rays have been instrumental in our 
understanding of structural biochemistry: the tremendous database of protein structures available today [1] has been solely determined using X-ray sources. X-ray sources have also proven indispensable to investigate material structure enabling engineering of materials from a fundamental understanding of its structure [2]. The more recent developments of X-ray sources are enabling simultaneous exquisite temporal and spatial resolution which promises further discoveries. Recent results have in particular revealed electronic rearrangements in proteins on the femtosecond time scale [3], femtosecond diffractive imaging of nanostructures [4] and catalysis dynamics on surfaces in real time [5].

The main sources of brilliant X-rays have so far been accelerator based large scale facilities such as synchrotrons and more recently free-electron lasers. These facilities provide ample amount of X-ray flux at photon energies in the hundreds of eV to multi-keV range [6]. While these large-scale facilities deliver $\mathrm{X}$-ray radiation suitable for a wide range of applications, their initial and operational costs are prohibitive and only a limited number of such facilities are available worldwide. Free-electron lasers in particular provide short pulses, high brightness X-rays that have enabled femtosecond serial crystallography [7], a new form of X-ray crystallography. To this day however, only a handful of FELs are available worldwide, therefore creating a bottleneck for fundamental and applied research.

Over the past two decades, the ultrafast optics community has developed, engineered and refined a tabletop approach to coherent X-ray generation via the high harmonic generation (HHG) process [8]. In addition to generating coherent $\mathrm{UV}$ to soft X-ray radiation, the HHG process allows extremely accurate synchronization with a laser system - a feature that is challenging using large scale facilities - and pulse durations as short as 43 attoseconds [9]. While these novel and accessible sources of X-rays have allowed a number of fundamental discoveries, they are currently limited in photon flux and scaling to a multi-keV photon energy source has yet to be demonstrated.

A third pathway toward tabletop, widely accessible X-ray sources has emerged recently relying on laser driven electron accelerators. The use of lasers to accelerate/undulate electrons rather than electro-magnets - as used in standard largescale facilities - allows downsizing the scale of the facilities and proportionally reduces cost of investment and ownership. Using direct electron acceleration in sub-mm scale accelerating structures, electron acceleration has been demonstrated but for limited charge [10], [11]. Plasma driven accelerators have shown record acceleration of electrons to 4.2 GeV, yet these facilities are bound to operate at low repetition rate and currently suffer from shot-to-shot reproducibility [12]. Another approach - that our group is pursuing - consists of accelerating electrons using $\mathrm{THz}$ frequency radiation generated from frequency down-converting state-of-the-art NIR lasers [13]. This approach is promising for accelerating pico-Coulomb electron bunches to the tens of $\mathrm{MeV}$ range. These electron beams can later interact with a high energy laser to generate $\mathrm{X}$-rays in an optical analogue to an undulator.

A particular challenge to our approach stems from generating temporally multi-cycle - equivalently, spectrally narrowband - THz pulses with multi-millijoule energy level. Indeed, the generation of such high energy $\mathrm{THz}$ pulses is bounded by both frequency down conversion efficiency and the state-of-the-art in both laser technology and nonlinear crystal development. The frequency down conversion is limited by the Manley-Rowe limit which states that the maximum efficiency for a three-wave mixing process to transfer energy from the pump wave to the idler wave is equal to the ratio of the idler photon energy to the pump photon energy. In the case of frequency down conversion from $1 \mu \mathrm{m}$ wavelength to $1 \mathrm{~mm}$ wavelength, the Manley Rowe limit is $0.1 \%$. On the other hand, the current state-of-the-art for picosecond lasers operating at several-hundreds of Hertz repetition rates is currently at $\sim 1 \mathrm{~J}$ pulse energy [14]. Therefore using the most up-to-date laser system reported for our application would hardly allow generating sufficient amount of $\mathrm{THz}$ to successfully power a THz-based linear accelerator. In addition to these limitations, further challenges arise when designing the nonlinear medium used for down conversion such as the damage threshold of organic nonlinear crystals at high repetition rate [15], the clear aperture and peak power handling limitations in periodically poled lithium Niobate [16] or the phase transitions in potassium Niobate [17]-[19].

In order to address these challenges, we are investigating two methods dubbed 'cascaded optical parametric amplification' [20], [21] and 'chirp-and-delay' [22] for the generation of multi-cycle THz radiation with NIR driving lasers. Both these methods promise efficiency beyond the Manley-Rowe limit and would therefore provide access to multi-cycle THz pulses with several millijoules of energy using currently available nonlinear crystals and driving lasers. Numerical simulations as well as preliminary experimental results on these two methods are reported in this paper. 


\section{DESCRIPTION OF METHODS}

In the cascaded optical parametric amplification approach shown in Figure 1 (left), a low energy narrowband pulse with central frequency $\omega_{\mathrm{s}}$ is collinearly and temporally overlapped with a high energy pulse with central frequency $\omega_{\mathrm{p}}=\omega_{\mathrm{s}}+$ $\omega_{\mathrm{i}}$ in a nonlinear medium where $\omega_{\mathrm{i}}$ is on the order of $500 \mathrm{GHz}$ at $1 \mu \mathrm{m}$ wavelength $\left(\Delta \lambda=\lambda_{\mathrm{p}}-\lambda_{\mathrm{s}}=1.6 \mathrm{~nm}\right)$. Owing to their narrow bandwidth these pulses are typically in the hundreds of picosecond duration. The nonlinear medium is chosen or engineered to phase-match the interaction at the chosen THz frequency $\omega_{\mathrm{i}}$. The optical pulses are chosen to have durations in the hundreds of picosecond duration in order to accommodate damage-free high energy pulses in a limited clear aperture since some nonlinear crystals - periodically poled lithium niobate in particular - are only available in limited aperture. Operating with long duration pulses also alleviates parasitic nonlinear effects and can be compensated for by increasing the length of the nonlinear medium. Increasing the length of the nonlinear medium typically reduces the phase-matchable bandwidth, a desirable feature when generating multi-cycle $\mathrm{THz}$ pulses.

The dynamics of the process first follows that of a standard optical parametric amplifier: the strong pump wave $\left(\omega_{\mathrm{p}}\right)$ amplifies the signal wave $\left(\omega_{\mathrm{s}}\right)$ and generates an idler wave $\left(\omega_{\mathrm{i}}\right)$. Owing to the momentum and energy conservation and to the spectral vicinity of the pump and signal waves, the idler wave can back-interact, phase-matched with the both the pump and signal waves via sum and difference frequency generation. Through this interaction, spectral side bands at $\omega_{1}=\omega_{\mathrm{s}}-\omega_{\mathrm{i}}$ and $\omega_{-1}=\omega_{\mathrm{p}}+\omega_{\mathrm{i}}$ are generated. The process can cascade resulting in a series of coherent spectral lines acting as numerous OPAs adding in phase to the THz radiation. Each of these OPAs can be operated at the Manley-Rowe limit and therefore the overall efficiency of the interaction exceeds the Manley-Rowe limit. Dispersion considerations must be taken into account to favor the difference frequency interaction between the THz and NIR radiations to ensure actual buildup of THz radiation [23]. Notice that alternatively, narrowband chirped pulse combinations with appropriate delays can be used, granted that the chirp rate of the pulses is similar in a so-called chirp-and-delay scheme [24].

In the chirp-and-delay approach shown in Figure 1 (right), one spectrally-chirped pulse is split into two identical replicas and recombined with a delay $\Delta t$ in a nonlinear medium designed to phase-match difference frequency generation to a THz frequency $\omega_{\mathrm{i}}$ between the two replicas. In this case, the spectral bandwidth $\Delta \omega$ should be greater than $\omega_{\mathrm{i}}$ and the pulse duration is adjusted by adjusting the spectral phase. The spectral phase needs however to be carefully controlled to maximize the efficiency of the process as discussed below. The value of the delay is set such that $\mathrm{C} . \Delta \mathrm{t}=\omega_{\mathrm{i}}$, where $\mathrm{C}$ is the chirp rate applied to the driving NIR pulses. The choice of using temporally stretched optical pulses with duration in the hundreds of picoseconds is motivated by the same considerations as in the cascaded optical parametric amplifier. The dynamics of the interaction is similar to that of the cascaded optical parametric amplifier yet the spectral signature in the NIR consists of broadening towards the long wavelength. In addition, care must be taken when applying spectral phase to the driving pulses to ensure optimum broadband overlap to fall within the phase matching bandwidth of the nonlinear medium.
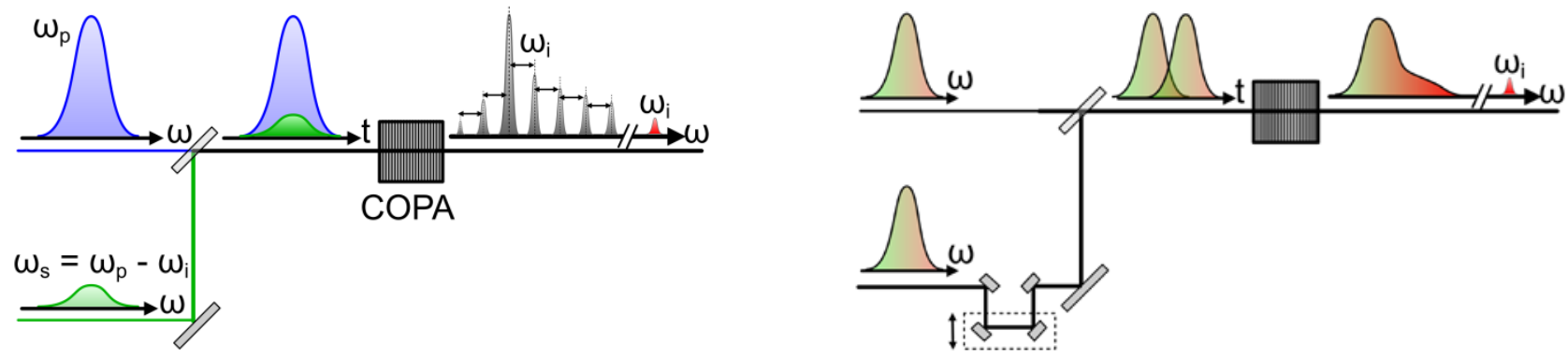

Figure 1. (Left) Schematic representation of the cascaded optical parametric amplification scheme; (right) schematic representation of the chirp-and-delay scheme. Both approaches promise THz radiation generation beyond the Manley-Rowe limit, therefore opening a pathway toward multi-millijoule, multi-cycle THz pulses using exciting laser technology and nonlinear gain media. 


\section{IMPLEMENTATION \& RESULTS}

\subsection{Cascaded optical parametric amplifier}

Our proof-of-principle implementation of the cascaded optical parametric amplifier concept (Figure 2) relied on optical pulses derived from a $6 \mathrm{~nm}$ bandwidth Yb:KYW regenerative amplifier delivering temporally-stretched pulses with up to $5 \mathrm{~mJ}$ output energy [25]. Approximately $1 \mathrm{~mJ}$ of energy was used for this experiment, the leftover being used for other experiments not described here. Of the $1 \mathrm{~mJ}$ available, $80 \%$ was sent to a 4-pass cryogenic Yb:YAG amplifier and further amplified to the $50 \mathrm{~mJ}$ range at $100 \mathrm{~Hz}$ repetition rate. Upon amplification, the pulses undergo severe spectral narrowing and emerge from the amplifier with a spectral width as narrow as $0.4 \mathrm{~nm}$ centered at $1029.5 \mathrm{~nm}$. The remaining $20 \%$ of the seed pulses were directed towards a narrowband ( $0.4 \mathrm{~nm}$ amplification bandwidth) OPA stage pumped by a fraction of the Yb:YAG cryogenic amplifier output - and optimized for operation at a central wavelength of $1031.25 \mathrm{~nm}$, thereby setting an offset frequency of $500 \mathrm{GHz}$ between the pump and signal beam. The pulse duration of the interacting pulses was measured to be 200 ps. The maximum energy used in the pump pulses was limited - by damage threshold and limited clear aperture of the cascaded OPA nonlinear crystal - to $15 \mathrm{~mJ}$ while the energy in the signal pulses was constant and set to $165 \mu \mathrm{J}$ at the cascaded OPA crystal. A set of periodically poled lithium niobate crystals with length of $0.5,1$ and $2 \mathrm{~cm}$ and a poling period of $212 \mu \mathrm{m}$ - phase-matching difference frequency generation from $1 \mu \mathrm{m}$ wavelength to $600 \mu \mathrm{m}$ wavelength (500 GHz THz frequency) - were used.

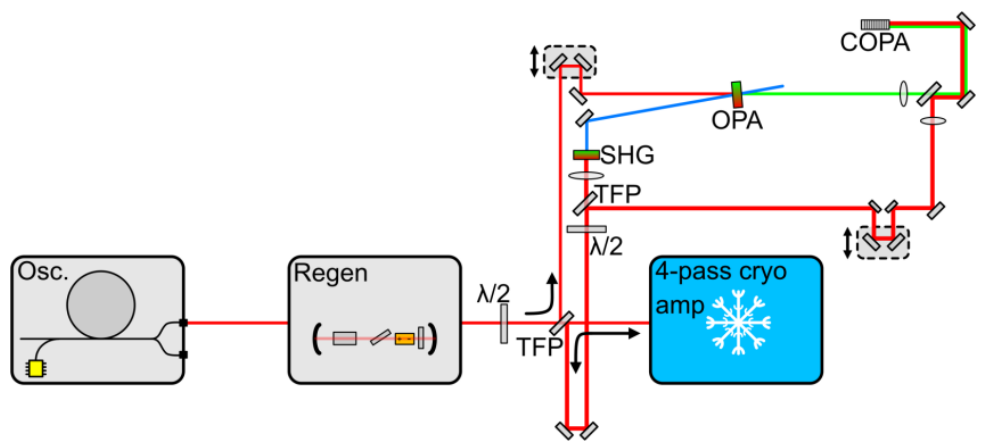

Figure 2. Layout of the optical setup generating the two interacting NIR narrowband optical pulses in the cascaded optical parametric amplifier.

Using the setup described above, we first characterized the spectral behavior of the cascaded optical parametric amplifier using a spectrum analyzer with a spectral resolution of $0.05 \mathrm{~nm}$ and a dynamic range greater than $50 \mathrm{~dB}$. We observed the onset of spectral cascading using a $0.5 \mathrm{~cm}$ crystal at fluences exceeding $0.5 \mathrm{~J} / \mathrm{cm}^{2}$. Resorting to longer crystals, the pump fluence threshold for observing spectral cascading lowered. In particular using the 2 cm crystal, we observed cascading at fluences as low as $0.2 \mathrm{~J} / \mathrm{cm}^{2}$. In addition, we observed preferential spectral shifting to longer wavelength which - as our numerical simulations predict [23] - originates from the dispersion of lithium niobate and is a signature of net THz generation (Figure 3).

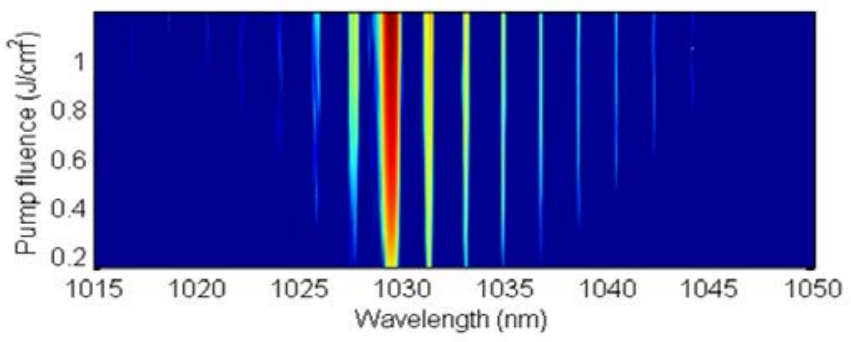

Figure 3. Measured cascaded spectrum as a function of pump fluence (arbitrary units logarithmic color scale) using a $2 \mathrm{~cm}$ long periodically poled lithium niobate crystal. The number of cascaded orders increases with crystal length and fluence and is preferentially red-shifted, indicating net generation of THz radiation. 
In order to further characterize the cascaded optical parametric amplifier, we directly measured the generated $\mathrm{THz}$ radiation using a calibrated pyroelectric detector. The NIR radiation was separated from the THz radiation using a 2" diameter off-axis parabola with a $\sim 5 \mathrm{~mm}$ hole in the middle. The off-axis parabola collected the highly diverging $\mathrm{THz}$ radiation while letting the mostly collimated NIR radiation through the central hole. We recorded the generated $\mathrm{THz}$ energy as a function of pump fluence for the three crystals available. The maximum amount of THz energy generated reached $160 \mathrm{~nJ}$, corresponding to an efficiency of $1.6 \times 10^{-5}$, well below the Manley-Rowe limit $(0.16 \%$ for $500 \mathrm{GHz}$ frequency radiation). We observe that increasing the crystal length did not provide any improvement on the $\mathrm{THz}$ yield (Figure 4 - left). In addition this is in apparent contradiction with the increasing amount of spectral cascading we observed when increasing the crystal length. We performed numerical simulations that successfully reproduced the experimental results which allowed identifying $\mathrm{THz}$ absorption in lithium niobate at room temperature (absorption coefficient at $500 \mathrm{GHz} \alpha_{\mathrm{RT}}=6 \mathrm{~cm}^{-1}$ ) as the cause for limiting the THz yield and also explain the absence of increase in THz energy when increasing crystal length. Changing the absorption coefficient at $500 \mathrm{GHz}$ to $\alpha_{\text {Cryo }}=2 \mathrm{~cm}^{-1}$ in the numerical simulations - an absorption coefficient value that can be experimentally obtained via cryogenic cooling of the lithium niobate crystal [26] -, we find that, the crystal length can be scaled up to $4 \mathrm{~cm}$ and efficiencies up to the percent level can be reached (Figure 4 - right).
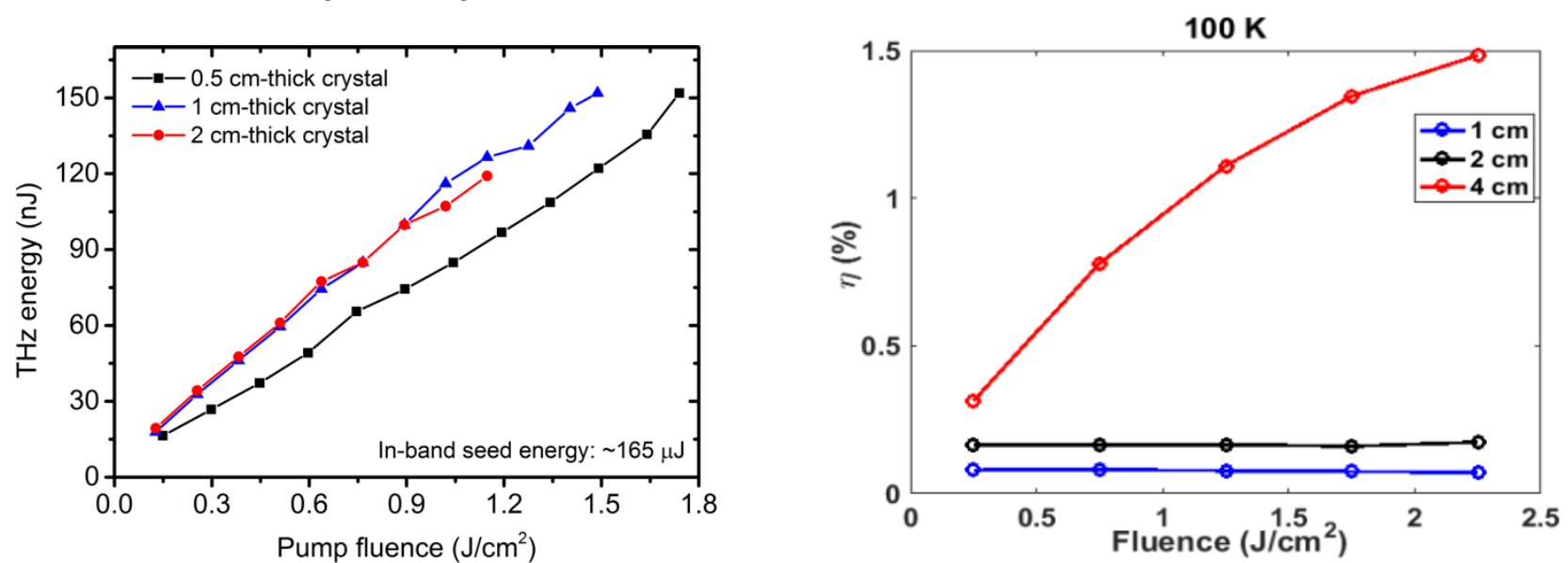

Figure 3. (Left) Measured THz energy versus pump fluence for $0.5 \mathrm{~cm}$ long, $1 \mathrm{~cm}$ long and $2 \mathrm{~cm}$ long crystals. The maximum efficiency reached $1.6 \times 10^{-3} \%$ for a $1 \mathrm{~cm}$ long crystal at $\sim 1.3 \mathrm{~J} / \mathrm{cm}^{2}$ fluence; (right) Computed THz generation efficiency for a $1 \mathrm{~cm}, 2$ $\mathrm{cm}$ and $4 \mathrm{~cm}$ long periodically poled lithium niobate crystal at cryogenic temperature. The use of cryogenic temperature reduces $\mathrm{THz}$ absorption yielding efficiencies at the percent level, beyond the Manley-Rowe limit.

\subsection{Chirp-and-delay}

Although chirp-and-delay experiments have previously been implemented in various formats, it has only recently been applied to periodically-poled crystals by our group [27], resulting in a new record in optically generated multi-cycle $\mathrm{THz}$ of $40 \mu \mathrm{J}$. This work, which was driven by high-energy Ti:Sapphire pulses at low repetition rates and employed cryogenic cooling to reduce $\mathrm{THz}$ absorption in periodically-poled Mg-doped lithium niobate (PP Mg:LN), has recently been extended by increasing laser pulse energies and crystal apertures as well as by tuning the spectral phase of the chirped pulse pair.

The spectral phase of the chirped pulses determines the instantaneous spectral content of the chirped pulses. To first order, the spectral phase in a Ti:Sapphire chirped-pulse amplifier (CPA) is quadratic, leading to a primarily linear temporal chirp. For the case of perfectly linear chirp, the instantaneous difference in local frequency between the two pulses is fixed, leading to a single delay that matches the frequency difference to the PPLN. However, due to the dispersion characteristics of standard CPA stretchers, the temporal chirp usually has a non-linear component that results in a local frequency difference that depends on time (Figure 4A). Since for a given inter-pulse delay only a narrow temporal slice of the chirped-pulse pair contains the appropriate spectral content (i.e. the correct local frequency 
difference) for DFG, the pump depletion associated with generation of $\mathrm{THz}$ waves was only observed at a particular wavelength. The spectral location of the pump depletion was dependent on delay (Figure 4B), confirming the presence of nonlinear chirp.
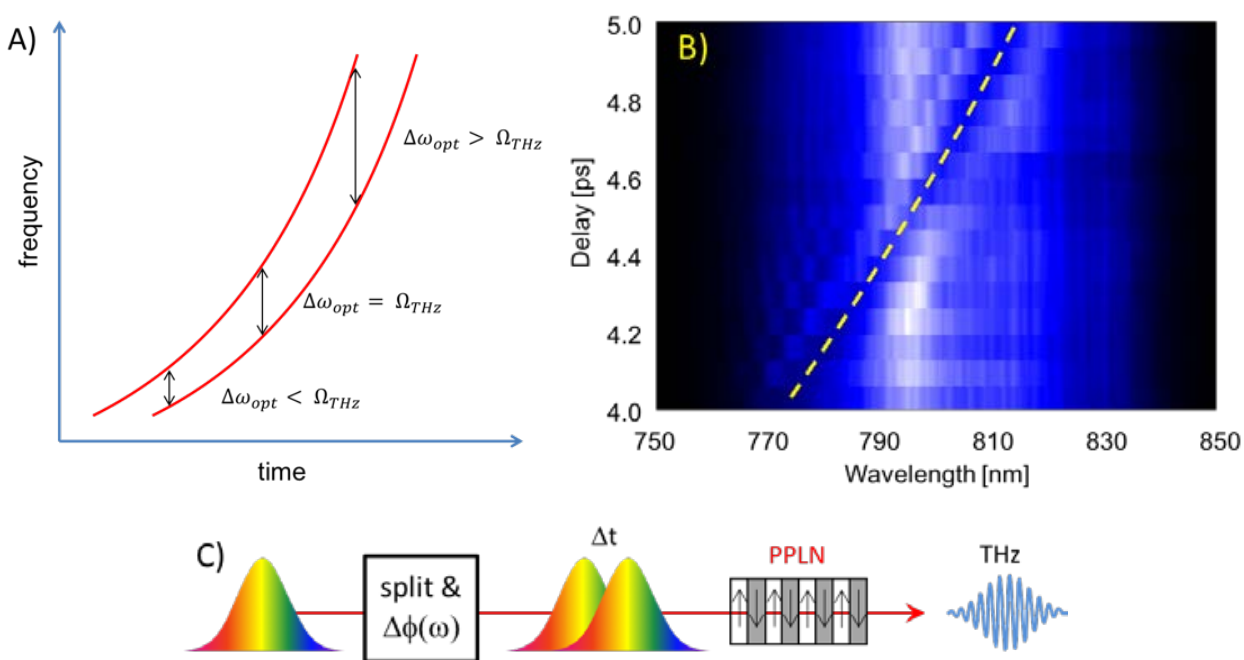

Figure 4. Chirp-and-delay with Ti:Sapphire pulses. A) Frequency-time diagram indicating the nonlinear dependence of local frequency on time in the chirped-pulse pair. B) Transmitted spectrum of chirped-pulse pair showing dependence of pump depletion on delay resulting from nonlinear temporal chirp. C) Schematic showing chirp-and-delay experimental setup.

In order to optimize the optical-to-THz conversion efficiency, the nonlinear chirp must be addressed to allow the entirety of the chirped-pulse pair to contribute to the conversion process. This was done by adjusting the spectral phase of the chirped pulses (Figure 4C), resulting in improvements in efficiency greater than a factor of five. Combining this efficiency increase with an increase in the applied energy made possible by the use of a large-aperture $(12 \times 15 \mathrm{~mm})$ PPMgLN [28], [29], a new record and order-of-magnitude increase in the output energy of the multi-cycle THz of over $400 \mu \mathrm{J}$ was achieved [30].

\section{CONCLUSIONS}

We are currently investigating two methods to dramatically improve the efficiency of multi-cycle $\mathrm{THz}$ radiation generation while accommodating the finite aperture size and damage threshold of nonlinear media. The experimental implementation of the first method has led to the successful observation of spectral cascading - and are in qualitative and quantitative agreement with our numerical model - and to the generation of $\mathrm{THz}$ radiation. The experimentally observed limited $\mathrm{THz}$ generation efficiency stems from $\mathrm{THz}$ absorption in lithium niobate at room temperature. Experiments are currently underway towards high $\mathrm{THz}$ generation efficiency using this method at reduced $\mathrm{THz}$ absorption via cryogenic cooling of the nonlinear medium. In parallel, we have investigated an alternative method using delayed high energy chirped pulses at NIR wavelength. A thorough investigation of the process and optimization of the spectral phase of the driving pulses has led to record $400 \mu \mathrm{J}$ multi-cycle THz pulses.

\section{ACKNOWLEDGMENTS}

The authors acknowledge funding from the European Research Council (ERC) under the European Union Seventh Framework Programme (FP7) (FP7/2007-2013)/ERC Grant (609920); European Regional Development Fund (ERDF), project ELI-Extreme Light Infrastructure-phase 2 (CZ.02.1.01/0.0/0.0/15_008/ 0000162). 


\section{REFERENCES}

[1] "Worldwide protein database.” [Online]. Available: http://www.wwpdb.org/.

[2] F. Lin, Y. Liu, X. Yu, L. Cheng, A. Sinjer, O. G. Shpyrko, H. L. Xin, N. Tamura, C. Tian, T.-C. Weng, X.-Q. Yang, Y. S. Meng, D. Nordlund, W. Yang, and M. M. Doeff, "Synchrotron X-ray Analytical Techniques for Studying Materials Electrochemistry in Rechargeable Batteries,” Chem. Rev., vol. 117, no. 21, pp. 13123-13186, 2017.

[3] F. Calegari, D. Ayuso, A. Trabattoni, L. Belshaw, S. De Camillis, S. Anumula, F. Frassetto, L. Poletto, A. Palacios, P. Decleva, J. B. Greenwood, F. Martin, and M. Nisoli, "Ultrafast electron dynamics in phenylalanine initiated by attosecond pulses,” Science (80-. )., vol. 346, no. 6207, pp. 336-339, 2014.

[4] H. N. Chapman, P. Fromme, A. Barty, T. A.White, R. A. Kirian, A. Aquila, M. S. Hunter, J. Schulz, D. P. DePonte, U. Weierstall, R. B. Doak, F. R. N. C. Maia, A. V. Martin, I. Schlichting, L. Lomb, N. Coppola, R. L. Shoeman, S. W. Epp, R. Hartmann, D. Rolles, A. Rudenko, L. Foucar, N. Kimmel, G. Weidenspointner, P. Holl, M. Liang, M. Barthelmess, C. Caleman, S. Boutet, M. J. Bogan, J. Krzywinski, C. Bostedt, S. Bajt, L. Grumpecht, B. Rudek, B. Erk, C. Schmidt, A. Hömke, C. Reich, D. Pietschner, L. Strüder, G. Hauser, H. Gorke, J. Ullrich, S. Herrmann, G. Schaller, F. Schopper, H. Soltau, K.-U. Kühnel, M. Messerscmidt, J. D. Bozek, S. P. Hau-Riege, M. Frank, C. Y. Hampton, T. G. Sierra, D. Starodub, G. J. Williams, J. Hadju, N. Timneanu, M. M. Seibert, J. Andreasson, A. Rocker, O. Jönsson, M. Svenda, S. Stern, K. Nass, R. Andritschke, C.-D. Scröter, F. Krasniqi, M. Bott, K. E. Schmidt, X. Wang, I. Grotjohann, J. M. Holton, T. R. M. Barends, R. Neutze, S. Marchesini, R. Fromme, S. Schorb, D. Rupp, M. Adolph, T. Gorkhover, I. Andersson, H. Hirsemann, G. Potdevin, H. Graafsma, B. Nilsson, and J. C. H. Spence, “Femtosecond X-ray protein nanocrystallography,” Nature, vol. 470, pp. 73-77, 2011.

[5] A. Nilsson, J. LaRue, H. Öberg, H. Ogasawara, M. Dell’Angela, M. Beye, H. Östrom, J. Gladh, J. K. Norskov, W. Wurth, F. Abild-Pedersen, and L. G. M. Pettersson, "Catalysis in real time using X-ray lasers,” Chem. Phys. Lett., vol. 675, pp. 145$173,2017$.

[6] “XFEL photon beam parameters.”[Online]. Available: http://xfel.desy.de/technical_information/photon_beam_parameter/.

[7] A. Meents, M. O. Wiedorn, V. Srajer, R. Henning, I. Sarrou, J. Bertgtholdt, M. Barthelmess, P. Y. A. Reinke, D. Dierksmeyer, A. Tolstikova, S. Schaible, M. Messerschmidt, C. M. Ogata, D. J. Kissick, M. H. Taft, D. J. Manstein, J. Lieske, D. Oberthuer, R. F. Fischetti, and H. N. Chapman, "Pink serial crystallography," Nat. Commun., vol. 8, p. 1281, 2017.

[8] P. B. Corkum, "Plasma Perspective on Strong-Field Multiphoton Ionization,” Phys. Rev. Lett., vol. 71, no. 13, pp. 19941997, 1993.

[9] T. Gaumnitz, A. Jain, Y. Pertot, M. Huppert, I. Jordan, F. Ardana-Lamas, and H. J. Wörner, "Streaking of 43-attosecond softX-ray pulses generated by a passively CEP-stable mid-infrared driver,” Opt. Express, vol. 25, no. 22, pp. 27506-27518, 2017.

[10] J. Breuer and P. Hommelhoff, "Laser-Based Acceleration of Nonrelativistic Electrons at a Dielectric Structure,” Phys. Rev. Lett., vol. 111, p. 134803, 2013.

[11] E. A. Peralta, K. Soong, R. J. England, E. R. Colby, Z. Wu, B. Montazeri, C. McGuiness, J. McNeur, K. J. Leedle, D. Waltz, E. B. Sozer, B. Cowan, B. Scwartz, G. Travish, and R. L. Byer, "Demonstration of electron acceleration in a laser-driven dielectric microstructure," Nature, vol. 503, pp. 91-94, 2013.

[12] W. P. Leemans, A. J. Gonsalves, H.-S. Mao, K. Nakamura, C. Benedetti, C. B. Schroeder, C. Toth, J. Daniels, D. E. Mittelberger, S. S. Bulanov, J.-L. Vay, C. G. R. Geddes, and E. Esarey, "Multi-GeV Electron Beams from CapillaryDischarge-Guided Subpetawatt Laser Pulses in the Self-Trapping Regime,” Phys. Rev. Lett., vol. 113, p. 245002, 2014.

[13] F. X. Kärtner, F. Ahr, A.-L. Calendron, H. Çankaya, S. Carbajo, G. Chang, G. Cirmi, K. Dörner, U. Dorda, A. Fallahi, A. Hartin, M. Hemmer, R. Hobbs, Y. Hua, W. R. Huang, R. Letrun, N. Matlis, V. Mazalova, O. D. Mücke, E. Nanni, W. Putnam, K. Ravi, F. Reichert, I. Sarrou, X. Wu, A. Yahaghi, H. Ye, L. Zapata, D. Zhang, C. Zhou, R. J. D. Miller, K. K. Berggren, H. Graafsma, A. Meents, R. W. Assmann, H. N. Chapman, and P. Fromme, "AXSIS: exploring the frontiers in attosecond X-ray science, imaging and spectroscopy,” Nucl. Instruments Methods Phys. Res. Sect. A Accel. Spectrometers, Detect. Assoc. Equip., vol. 829, pp. 24-29, 2016.

[14] C. M. Baumgarten, B. A. Reagan, M. Pedicone, H. Bravo, L. Yin, H. Wang, M. Woolston, B. Carr, C. Menoni, and J. Rocca, "Demonstration of a Compact $500 \mathrm{~Hz}$ Repetition Rate Joule-Level Chirped Pulse Amplification Laser," in Conference on Laser and Electro-Optics, 2016, p. paper STu3M.3.

[15] S. S. Dhillon, M. S. Vitiello, E. H. Linfield, A. G. Davies, M. C. Hoffmann, J. Booske, C. Paolini, M. Gensch, P. Weightman, and G. P. Williams, “The 2017 terahertz science and technology roadmap,” J. Phys. D. Appl. Phys., vol. 50, no. 4, pp. 1-49, 2017.

[16] H. Ishizuki and T. Taira, "High-energy quasi-phase-matched optical parametric oscillation in a periodically poled MgO:LiNbO3 device with a 5 mm x 5 mm aperture,” Opt. Lett., vol. 30, no. 21, pp. 2918-2921, 2005.

[17] M. Baudisch, M. Hemmer, H. Pires, and J. Biegert, "Performance of MgO:PPLN, KTA, and KNbO3 for mid-wave infrared broadband parametic amplification at high average power,” Opt. Lett., vol. 39, no. 20, pp. 5802-5805, 2014.

[18] L. Xian-jie, Z. Xing-kui, and X. Xiu-ying, "Domain Patterns and Phase Transitions in Ferroelectric Crystal KNbO3,” Chinese Phys. Lett., vol. 13, no. 8, p. 621, 1996.

[19] M. M. Shamim and T. Ishidate, "Anomalous mode coupling and phase transition of KNbO3 under high pressure," Solid State Commun., vol. 113, no. 12, pp. 713-717, 2000. 
[20] A. G. Stepanov, A. A. Mel'nikov, V. O. Kompanets, and S. V. Chekalin, "Spectral modification of femtosecond laser pulses in the process of highly efficient generation of terahertz radiation via optical rectification,” JETP Lett., vol. 85, no. 5, pp. 227-230, 2007.

[21] M. Cronin-Golomb, “Cascaded nonlinear difference-frequency generation of enhanced terahertz wave production,” Opt. Lett., vol. 29, no. 17, pp. 2046-2048, 2004.

[22] A. S. Weling, B. B. Hu, N. M. Froberg, and D. H. Auston, "Generation of tunable narrow-band THz radiation from large aperture photoconducting antennas,” Appl. Phys. Lett., vol. 64, no. 2, pp. 137-139, 1994.

[23] K. Ravi, M. Hemmer, G. Cirmi, F. Reichert, D. N. Schimpf, O. D. Mücke, and F. X. Kärtner, "Cascaded parametric amplification for highly efficient terahertz generation,” Opt. Lett., vol. 41, no. 16, pp. 3806-3809, 2016.

[24] Z. Chen, X. Zhou, C. A. Werley, and K. A. Nelson, "Generation of high power tunable multicycle teraherz pulses,” Appl. Phys. Lett., vol. 99, p. 071102, 2011.

[25] G. Cirmi, M. Hemmer, K. Ravi, F. Reichert, L. E. Zapata, A.-L. Calendron, H. Cankaya, F. Ahr, O. D. Mücke, N. H. Matlis, and F. X. Kärtner, “Cascaded second-order processes for the efficient generation of narrowband terahertz radiation,” J. Phys. B At. Mol. Opt. Phys., vol. 50, no. 4, 2017.

[26] X. Wu, C. Zhou, W. R. Huang, F. Ahr, and F. X. Kärtner, "Temperature dependent refractive index and absorption coefficient of congruent lithium niobate crystals in the terahertz range,” Opt. Express, vol. 23, no. 23, pp. 29729-29737, 2015.

[27] F. Ahr, S. W. Jolly, N. H. Matlis, S. Carbajo, T. Kroh, K. Ravi, D. N. Schimpf, J. Schulte, H. Ishizuki, T. Taira, A. R. Maier, and F. X. Kärtner, "Narrowband terahertz generation with chirped-and-delayed laser pulses in periodically poled lithium niobate,” Opt. Lett., vol. 42, no. 11, pp. 2118-2121, 2017.

[28] H. Ishizuki and T. Taira, "Improvement of laser-beam distortion in large-aperture PPMgLN device by using X-axis Czochralski-grown crystal,” Opt. Express, vol. 22, no. 16, pp. 19668-19673, 2014.

[29] H. Ishizuki and T. Taira, "Half-joule output optical parametric oscillation by using 10-mm-thick periodically poled Mg-doped congruent LiNbO3,” Opt. Express, vol. 20, no. 18, pp. 20002-20010, 2012.

[30] S. W. Jolly, F. Ahr, N. H. Matlis, V. Leroux, T. Eichner, K. Ravi, H. Ishizuki, T. Taira, and F. X. Kärtner, "Millijoule-scale narrowband terahertz pulses via phase manipulation of pump laser pulses” in preparation, 2018. 